\title{
Byzantinische Spitalgeschichte
}

\author{
Von Urs Benno Birchler-Argyros
}

\begin{abstract}
Timothy S.Miller: The Birth of the Hospital in the Byzantine Empire. Bulletin of the history of Medicine. New Series No. 10. The Johns Hopkins University Press, Baltimore/London 1985.
\end{abstract}

Das Thema «byzantinische Spitalgeschichte» erfreut sich in den letzten Jahren erneuter Aktualität. Schon zu Beginn des Jahrhunderts (1913) hat K.Sudhoff die Medizinhistoriker darauf hingewiesen, und am ersten internationalen Kongreß für Medizingeschichte in Anvers (1920) wurde es von E. Jeanselme und L. Oeconomos bearbeitet. Danach trat eine Ruhepause ein, bis das Sujet, diesmal von Byzantinisten, wiederaufgegriffen wurde mit A.Philippsborn (1961), D.J.Constantelos (1968), P. Gautier (1974) und R. Volk (1983), um nur die wichtigsten zu nennen. Mit Miller fand es nun als Supplementum Eingang in das Bulletin of the history of medicine.

Millers Arbeit liest sich wie ein Roman. Seine Ergebnisse lassen sich kurz folgendermaßen zusammenfassen (Epilogue): Seit Beginn des Reiches hat es in Byzanz hochorganisierte Krankenhäuser gegeben. Ein Spital gehörte genauso zu einer Polis wie das Aquädukt, Hippodrom oder das Theater. Seit Justinian seien die aus der Antike bekannten Stadtärzte [archiatroi] zu Krankenhausbeamten umgewandelt worden, was zur Entwicklung eines hierarchisch gegliederten Ärztecorps geführt habe. Ärzte seien seither nicht mehr in Privatpraxen ausgebildet worden wie im Altertum, sondern in Krankenhäusern, die mit Patientenstationen, Polikliniken, Schulen, Bibliotheken und Skriptorien versehen waren. Dank den Spitälern sei in Byzanz eine «soziale Medizin» praktiziert worden, da die Ärzte nicht mehr von den Patienten bezahlt wurden, sondern vom Spital besoldet waren. Damit sei der Bevölkerung aller Schichten dieselbe Qualität der medizinischen Versorgung zugesichert worden. Kurz, laut Miller sind byzantinische Krankenhäuser denjenigen moderner Staaten zu vergleichen.

Seine recht erstaunlichen Schlüsse versucht Miller in neun Kapiteln seines Buches zu untermauern. Nach einer Einleitung bespricht er die Statuten des Pantokrator-Krankenhauses, zur Zeit das quellenmäßig best- 
bekannte oströmische Spital aus dem Jahre 1136. Die Kapitel 3-7 dienen dem Nachweis, daß der Pantokrator nicht einen Einzelfall darstellte, sondern $\mathrm{da} \beta$ es ähnliche Institutionen seit dem 6. Jahrhundert gegeben habe. Neben zahlreichen Indizien, die der Autor geschickt zu kombinieren vermag, gelingt es ihm, selbst schwer überschaubare Strömungen wie den Arianismus oder Hesychasmus als logische Vorbedingungen in die Entwicklung des Spitalwesens einzubauen. Aus den Hinweisen zur Frühzeit des Byzantinischen Reiches stechen zwei Quellen hervor: die Miracula Artemisii und die Justinianische Gesetzgebung. Auf die Wichtigkeit der Miracula für die Hospitalgeschichte hatte schon H. J. Magoulias hingewiesen (1964).

Mit der Annahme, daß Kaiser Justinian den Archiatros in einen Spitalarzt umgewandelt habe, sei quasi die Entwicklung einer staatlich organisierten Krankenhausmedizin eingeleitet worden, die nach dem Autor ihre Blütezeit unter der Komnenendynastie fand.

Anschaulich versucht Miller im 8. Kapitel ein byzantinisches Spital im Alltag nachzuzeichnen; Patienten aller sozialen Schichten, die in eigenen Betten in ikonen- und mosaikgeschmückten Räumen liegen; ein Ärzteteam, das sie in Anwesenheit von Studenten genau untersucht und hippokratische Medizin betreibt; Polikliniken zur Behandlung ambulanter Patienten; Spitalbibliotheken, von Ärzten und Studenten besucht, usw.

Das 10. Kapitel ist den Jahren nach 1204 gewidmet bis zum Fall des Reiches in die Hände der Türken, eine Zeitepoche, in der vom Autor generell der Niedergang der oströmischen Spitalentwicklung festgestellt wird.

Millers Arbeit ist ideenreich, doch er ist wie geblendet von Pantokrator, und seine Interpretation der Quellen ist stark von modernen Vorstellungen behaftet. Wir wollen dies hier anhand von zwei Beispielen zeigen: dem medizinischen Unterricht und der «sozialen Medizin».

Miller vermittelt den Eindruck, als hätte es in byzantinischen Spitälern seit den Anfängen eine medizinische Ausbildung im modernen Sinn gegeben. Mit Sicherheit kann eine Schule an einem Krankenhaus erst am Pantokrator nachgewiesen werden, ohne daß wir Einzelheiten über den Unterricht erfahren. Vermutlich wurde dieser im mittelalterlich-scholastischen Sinne erteilt, jedenfalls haben wir keine Beweise für eine grundlegende Neuerung im Unterrichtswesen. Seit dem Alterum haben Ärzte und deren Lehrlinge Kranke an ihren Betten besucht, dennoch sprechen wir von einer «klinischen» Medizin erst in der Neuzeit. Der Beweis für eine so epochemachende Neuerung müßte in der zeitgenössischen medizinischen Literatur gefunden werden. Miller deutet zwar darauf hin, wenn er von neuen chirurgischen 
Techniken, ja von einer eigentlichen pathologischen Anatomie mit Sektionen in der byzantinischen Medizin spricht. Die Quellen darüber sind jedoch ausnahmslos nichtmedizinischer Natur (Hagiographen, Poeten, Geschichtsschreiber), spärlich und zur Zeit wenig überzeugend. Wenn das Pantokratorstatut auch verblüffende Parallelen zur heutigen Zeit aufweist, gilt dennoch das Sprichwort: «Wenn zwei dasselbe tun, ist es nicht das gleiche.» Wenn die jungen Ärzte im Pantokrator neben oder nach ihrer theoretischen Ausbildung eine Laufbahn im Spital absolvieren, so beweist dies zwar eine klar strukturierte praktische Ausbildung, nicht aber eo ipso einen Unterricht im heutigen Sinn!

Als zweiter Punkt sei die von Miller gelobte «soziale Medizin» der Byzantiner diskutiert. Mit Recht unterstreicht der Autor die politischen Motive, die zur Entwicklung des Krankenhauswesens geführt haben. Wie die Arena zum alten, so gehörte die Wohlfahrtsanstalt zum Stadtbild des neuen Rom. Kaiser, Klöster und reiche Privatpersonen errichteten Spitäler als Denkmäler ihrer christlichen Gesinnung. Von einer gezielten «Gesundheitspolitik» kann aber nicht gesprochen werden. Selbst der Pantokrator wurde aus persönlichen Motiven errichtet. Der Zweck der Gründung war es, eine Schar von Elenden zu versammeln, die Gott für das Seelenheil des Stifters anflehen sollte. Man darf daher nicht von einer gezielten Gesundheitspolitik, geschweige denn von einer allgemeinen medizinischen Versorgung der Bevölkerung sprechen. Alle bisher bekannten byzantinischen Spitäler sind privaten Einzelinitiativen entsprungen und hätten schon rein zahlenmäßig niemals genügt, eine Großstadt wie Konstantinopel auch nur annähernd zu versorgen.

Sicher kam dem Krankenhaus in Byzanz eine viel größere Wichtigkeit zu, als bisher angenommen wurde. Schon früh hat sich das Krankenhaus aus der christlichen Wohlfahrtsanstalt herauskristallisiert. In der Organisation dieser Institution haben die Byzantiner Pionierarbeit geleistet. Dabei wurde die medizinische Tradition der Antike mit diesen Institutionen verbunden, und es ist bezeichnend, daß es in Byzanz nie eine «Mönchsmedizin» wie im Westen gegeben hat. Dies mag die Voraussetzungen geschaffen haben, die dann anderswo (wie z. B. in Salerno oder Padua, beide unter indirektem byzantinischem Einfluß über Süditalien oder Venedig) zu weiteren Entwicklungen geführt haben. Zu Recht hat Miller das Thema der byzantinischen Spitalgeschichte auf den «medizinhistorischen Diskussionstisch» gelegt, doch muß man sich bewußt bleiben, daß er in gewagter Weise beinahe hinter jedem oströmischen Spital einen Pantokrator vermutet! 
Wichtige Anstöße gibt Miller im 9. Kapitel über "Hospitals and medical Literature". A. Kouzis (1927) und E. Jeansleme (1930) haben bereits auf diese Spitalliteratur hingewiesen. Miller hat diesen Impuls wieder aufgegriffen und durch neue Hinweise erweitert. Dieses Gebiet ist noch wenig erforscht und könnte interessante Resultate zeitigen!

Millers Arbeit ist zwar voller spannender Theorien, doch schießt der Autor weit über die Wirklichkeit hinaus. Dem an der Realität der byzantinischen Krankenhäuser interessierten Leser sei deshalb R. Volks Arbeit über das Gesundheitswesen und Wohltätigkeit im Spiegel der byzantinischen Klostertypika empfohlen (Miscellanea byzantinea Monacensia No.28, München 1983). Volks Arbeit ist an Byzantinisten gerichtet und gibt Textstellen nur auf Griechisch wieder, doch sind seine Erläuterungen ausgiebig und bleiben im Rahmen der Fakten. An dieser Stelle sei auch die Arbeit von K. MentzouMeimare (1982) erwähnt, die uns zeigt, daß Wohlfahrtsanstalten auch in der Provinz des Byzantinischen Reiches verbreitet waren (Provinzielle Wohlfahrtsanstalten bis zum Ende des Bilderstreites, Byzantina, Band 11, Thessaloniki 1982, griech.).

\section{Summary}

Review of different papers on the history of Byzantine hospitals.

Dr. med. et med.dent. Urs Benno Birchler-Argyros

2 , rue de la Pépinière

CH-1201 Genève 